\title{
Journal of Orthopaedic Science and Research
}

Open Access

Case Report

\section{Non Metastatic Rhabdomyosarcoma in Children and Adolescents: Prognostic Factors and Survival}

\author{
Feryel Letaief-Ksontini ${ }^{1 *}$, Mouna Ayadi' ${ }^{1}$, Azza Gabsi ${ }^{1}$, Safia Yahiaoui ${ }^{2}$, Houcine Magherbi ${ }^{3}$, Amina \\ Mokrani $^{1}$, Khedija Meddeb ${ }^{1}$ \\ ${ }^{1}$ Associate Professor, Medical Oncology Department, Faculty of Medicine of Tunis, Salah Azaiez Institute, El \\ Manar University, Tunis, Tunisia \\ ${ }^{2}$ Associate Professor, Radiotherapy Department, Faculty of Medicine of Tunis, Salah Azaiez Institute, El Manar \\ University, Tunis, Tunisia \\ ${ }^{3}$ Associate Professor, Surgery Department, Faculty of Medicine of Tunis, La Rabta Hospital, El Manar \\ University, Tunis, Tunisia \\ *Corresponding Author: Feryel Letaief Ksontini, MD, Assistant Professor, Department of Medical Oncology, \\ Salah Azaiez Institute, Tunis, Tunisia and University of Tunis el Manar Faculty of Medicine of Tunis, Tunisia \\ Email: feryel.ksontini@gmail.com
}

Received Date: 08-09-2021; Accepted Date: 30-09-2021; Published Date: 07-10-2021

Copyright $^{\circledR} 2021$ by Ksontini FL, et al. All rights reserved. This is an open access article distributed under the terms of the Creative Commons Attribution License, which permits unrestricted use, distribution and reproduction in any medium, provided the original author and source are credited.

\begin{abstract}
Background: Rhabdomyosarcoma (RMS) is the most common softtissue tumor in childhood. We aimed to study the prognostic factors of non-metastatic RMS in Tunisian paediatric patients.

Methods: We reviewed data of paediatric patients (aged $<18$ years), with histologically confirmed RMS treated in Salah Azaiez Institute for a non-metastatic RMS during 20 years. Prognostic factors were studied and survival data analysed.

Results: 75 patients were included (42 males and 33 females). Embryonal RMS was the most common histologic type (72\%) followed by alveolar (21\%) and pleomorphic (1\%). The most frequently affected sites were head and neck (43\%) and genito-urinary (28\%). 5-year OS and DFS were $50 \%$ and $26 \%$, respectively. By univariate analysis, DFS was significantly correlated to chemotherapy, Radiotherapy (RT) and post-surgical RT with $\mathrm{p}=0.02,0.003$ and 0.01 , respectively. No factor was significant in multivariate analysis. By univariate analysis, 5-year

Ksontini FL | Volume 2; Issue 3 (2021) | JOSR-2(3)-019 | Case Report

Citation: Ksontini FL, et al. Non Metastatic Rhabdomyosarcoma in Children and Adolescents:

Prognostic Factors and Survival. J Ortho Sci Res. 2021;2(3):1-10.

DOI: http://dx.doi.org/10.46889/JOSR.2021.2302
\end{abstract}


OS was significantly and adversely influenced by 4 factors: tumour size $>4 \mathrm{~cm}$, non-alveolar RMS, positive regional nodes and para-meningeal location, with $\mathrm{p}=0.050,0.05,0.04$ and 0.04 , respectively. RT and postsurgical $\mathrm{RT}$ were associated with a good prognosis in OS $\mathrm{p}=0.009$ and 0.05 , respectively. By multivariate analysis, OS was strongly correlated to radiotherapy $\mathrm{p}=0.03$, Odds Ratio (OR) 3.1, (IC) 95\% [1.05-9.3] and para-meningeal site $\mathrm{p}=0.04$, (OR) 0.3, (IC) $95 \%$ [0.1-0.9].

Conclusions: This study showed that tumor size, histological type, tumor location, node involvement, t CT and RT were prognostic factors for OS and PFS. However, survival remains poor. So we should improve it by encouraging clinical research.

\section{Keywords}

Pediatric Rhabdomyosarcoma; Prognostic Factors; Survival; Surgery; Chemotherapy; Radiotherapy

\section{Introduction}

Rhabdomyosarcoma is the most common soft tissue tumor in childhood [1]. During the last 30 years, the introduction of multimodal therapy has resulted in a significant improvements in survival, with a cure rate of approximately $70 \%$ for patients with localized disease [1-3].

Several trials from Collaborative pediatric groups such as the Intergroup Rhabdomyosarcoma Study Group (IRSG), have revolutionized the therapeutic methods for this sarcoma [3]. Based on the conclusions of these studies, multimodal treatment regimens, involving surgery, chemotherapy and/or radiation, are decided by tumor staging (based on tumor primary site, tumor size, the presence or not of regional lymph node involvement and of distant metastasis), grouping (defined by the amount of residual tumorrafter initial surgery) and the histologiccsubtype of the tumor [3].

\section{Patients and Methods}

The overall study population consisted of 75 children aged $<$ or $=18$ years, with non-metastatic RMS treated between 1994 and 2016. Patients with isolated regional lymph node involvement were not considered to have metastatic disease. All patients had received histological confirmation of tumor. All patients received conventional multiagent chemotherapy based on alkylating agents (cyclophosphamide or ifosfamide), vincristine and dactinomycin. Some patients received other drugs, depending on the research group and specific protocol. Patient 
subsets with risk of treatment failure were identified on IRS post-surgical groups (Table 1 and 2).

Pre-treatment stratification IRSG (Intergroup Rhabdomyosarcoma Study Group) before 2005 was detailed in the Table 1 and 2. This classification was suitable for the patients treated between 1994-2004.The EpssG (European Paediatric Soft Tissue Sarcoma Study Group) had conducted a new risk group stratification in the protocol RMS 2005 (Table 3). So our patients diagnosed between 2005 and 2016 were classified by this protocol.

\begin{tabular}{|c|c|}
\hline Clinical Group & Definition \\
\hline Group I & Localized disease, completely resected \\
\hline \multirow{2}{*}{ Group II } & a: Grossly resected tumor with microscopic residual disease \\
\cline { 2 - 2 } & $\begin{array}{c}\text { b: Regional disease with involved nodes, completely resected with no } \\
\text { microscopic residual disease }\end{array}$ \\
\cline { 2 - 2 } & $\begin{array}{c}\text { c: Regional disease with involved nodes, grossaly resected but with } \\
\text { evidence of microscopic residual disease and/or histologic involvement } \\
\text { of the most distal regional mode (from the primary site) in the dissection }\end{array}$ \\
\hline Group III & Incomplete resection with gross residual disease \\
\hline Group IV & Distant metastatic disease present at onset \\
\hline
\end{tabular}

Table 1: Surgical-Pathologic grouping system of the intergroup Rhabdomyosarcoma study.

\begin{tabular}{|c|c|c|c|c|c|}
\hline Stage & Site & Statut $\mathbf{T}$ & Size & $\mathbf{N}$ & $\mathbf{M}$ \\
\hline 1 & Favourable & T1 or T2 & a or b & N0, N1 or Nx & M0 \\
\hline 2 & Unfavourable & T1 or T2 & $a$ & N0 or Nx & M0 \\
\hline 3 & Unfavourable & T1 or T2 & $a$ & N1 & M0 \\
\cline { 4 - 6 } & & & b & N0, N1 or Nx & M0 \\
\hline 4 & Favourable or unfavourable & T1 or T2 & a or b & N0 or N1 & M1 \\
\hline
\end{tabular}

Table 2: Pre-treatment IRSG staging system. 
The pre-treatment patient characteristics considered as prognostic factors were evaluated with univariate analysis using the Kaplan-Meier method to calculate survival probabilities for DFS and OS at 5 years [4]. The log-rank test compared survival differences. Associations among variables were assessed with the chi 2 test. Multivariate analysis was conducted during the Cox proportional hazards regression method. All calculations were performed with SPSS (statistical package for social sciences) version 20 .

\section{Results}

\section{Patient Characteristics}

The characteristics of the 75 patients included in the analysis are listed in Table 4. Median age at diagnosis was 8 years, but most patients $(71 \%)$ were younger than 10 years of age. Male patients outnumbered female patients (Sex ratio=1.27). Embryonal RMS was the most common histologic type (72\%) followed by alveolar (21\%) and pleomorphic (1\%). The most frequently affected sites were head and neck (43\%) and genito-urinary (28\%). As per Intergroup Rhabdomyosarcoma Study Group (IRSG) site classification, just over half of tumors were located at favorable prognostic site (orbit; non para meninge all head and neck; genitourinary tract except kidney, bladder and prostate; biliary tract).

\section{Survival}

For the entire cohort, the mean follow-up of survivors was 300 months, with a range of 1 month to 17.1 years. Estimated 5 year OS and DFS for all patients were $550 \%$ and $126 \%$, respectively. There were no differences in survival by age or sex. Analysis by histology type revealed that alveolar had the best overall survival (5-year OS =90\%, P =0.05) (Fig. 1). Patients with tumors $<$ or $=4 \mathrm{~cm}$ in size and without any lymph node involvement had better survival (5-year OS $=65 \%$, 60\%, respectively) (Fig. 1). The tumor site failed to be significant but only unfavourable para meningeal RMS was associated to a worst prognosis (Fig. 1).Surgical resection wasn't associated with improved survival (5-y OS: 53\% versus 50\% for no surgery, $\mathrm{p}=0.5)$. Overall, radiation therapy mainly post-surgical RT was associated with an overall 5year survival improvement ( $\mathrm{p}=0.009$ ) (Fig. 1). However, Chemotherapy (CT) didn't improve the overall 5 year survival ( $45 \%$ versus $28 \%$ without $\mathrm{CT}, \mathrm{p}=0.07$ ). The Table 5 shows the different protocols of neoadjuvant CT.

The predictive factors in DFS by univariate analysis were: chemotherapy, radiotherapy and post-surgical RT with p $0.02,0.003$ and 0.01 , respectively. 


\section{Multivariate Analysis}

Results of multivariate analysis using the Cox regression model revealed that OS was strongly correlated to radiotherapy $\mathrm{p}=0.03$, Odds Ratio (OR) 3.1, Confidence Interval (IC) $95 \%$ [1.059.3] and para meningeal site $\mathrm{p}=0.04$, Odds Ratio (OR) 0.3 , Confidence Interval (IC) $95 \%$ [0.1$0.9]$. We did not found any factor significantly correlated to the DFS.

\begin{tabular}{|c|c|c|c|c|c|}
\hline \multicolumn{2}{|c|}{ Characteristics } & Number & $\%$ of Total & 5-year OS & Log Rank Test (p) \\
\hline \multirow[t]{2}{*}{ Sex } & Male & 42 & 56 & $42 \%$ & \multirow[t]{2}{*}{0.7} \\
\hline & Female & 33 & 44 & $46.20 \%$ & \\
\hline \multirow[t]{3}{*}{ Age, Years } & $<5$ & 31 & 42 & $47.50 \%$ & \multirow[t]{3}{*}{0.14} \\
\hline & 10-May & 22 & 29 & $27.60 \%$ & \\
\hline & 18-Nov & 22 & 29 & $38.60 \%$ & \\
\hline \multirow[t]{8}{*}{ Primary Site } & Orbit & 11 & 14.7 & & \multirow[t]{2}{*}{0.7} \\
\hline & $\begin{array}{l}\text { Non-PM } \\
\text { head and } \\
\text { neck }\end{array}$ & 14 & 18.7 & & \\
\hline & $\mathrm{PM}$ & 7 & 9.4 & $20 \%$ & 0.041 \\
\hline & $\begin{array}{c}\text { GU(bladder/p } \\
\text { rostate })\end{array}$ & 4 & 5.4 & & \multirow[t]{5}{*}{0.7} \\
\hline & $\begin{array}{c}\text { GU(non- } \\
\text { bladder/prost } \\
\text { ate) }\end{array}$ & 17 & 22.6 & & \\
\hline & Limbs & 5 & 6.6 & & \\
\hline & other & 17 & 22.6 & & \\
\hline & No primary & 0 & 0 & & \\
\hline \multirow[t]{2}{*}{ Primary Site } & Favourable & 42 & 56 & $48 \%$ & \multirow[t]{2}{*}{0.7} \\
\hline & Unfavourable & 33 & 44 & $40 \%$ & \\
\hline \multirow{3}{*}{$\begin{array}{l}\text { Regional Nodal } \\
\text { Status }\end{array}$} & No & 50 & 66.7 & $60 \%$ & \multirow[t]{3}{*}{0.041} \\
\hline & Yes & 21 & 28 & $40 \%$ & \\
\hline & Unknown & 4 & 5.3 & - & \\
\hline \multirow[t]{2}{*}{ Size } & $<$ or $=4 \mathrm{~cm}$ & 18 & 24 & $65 \%$ & \multirow[t]{2}{*}{0.05} \\
\hline & $>4 \mathrm{~cm}$ & 55 & 73.3 & $42 \%$ & \\
\hline \multirow[t]{2}{*}{ Pathology } & Alveolar & 16 & 78.6 & $90 \%$ & \multirow[t]{2}{*}{0.05} \\
\hline & non alveolar & 59 & 21.4 & $38 \%$ & \\
\hline \multirow{2}{*}{$\begin{array}{l}\text { Period of } \\
\text { Treatment }\end{array}$} & before 2005 & 29 & 38.6 & $40.50 \%$ & \multirow[t]{2}{*}{0.14} \\
\hline & after2005 & 46 & 61.4 & $53 \%$ & \\
\hline Chemotherapy & yes & 70 & 93 & $45 \%$ & 0.07 \\
\hline
\end{tabular}




\begin{tabular}{|c|c|c|c|c|c|}
\hline \multirow{3}{*}{ Radiation } & no & 5 & 7 & $28 \%$ & \\
\cline { 2 - 5 } & yes & 36 & 48 & $71 \%$ & 0.009 \\
\hline \multirow{2}{*}{ Surgery } & no & 39 & 52 & $31 \%$ & \\
\cline { 2 - 5 } & yes & 37 & 49.3 & $53 \%$ & 0.5 \\
\hline
\end{tabular}

Table 4: Patient characteristics.

\begin{tabular}{|c|c|}
\hline Protocol & Number of Patients \\
\hline MMT89 & 6 \\
\hline MMT95 & 18 \\
\hline IVADO (RMS 2005) & 12 \\
\hline IVA (RMS 2005) & 7 \\
\hline VAC-VAD & 2 \\
\hline OTHERS & 3 \\
\hline
\end{tabular}

Table 5: Neoadjuvant chemotherapy protocols.
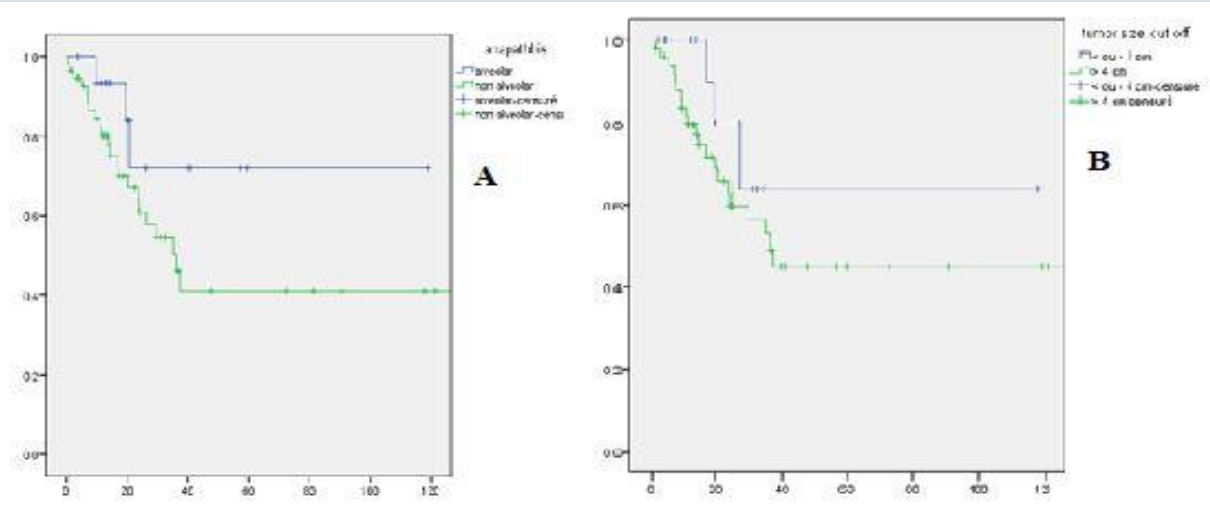

B
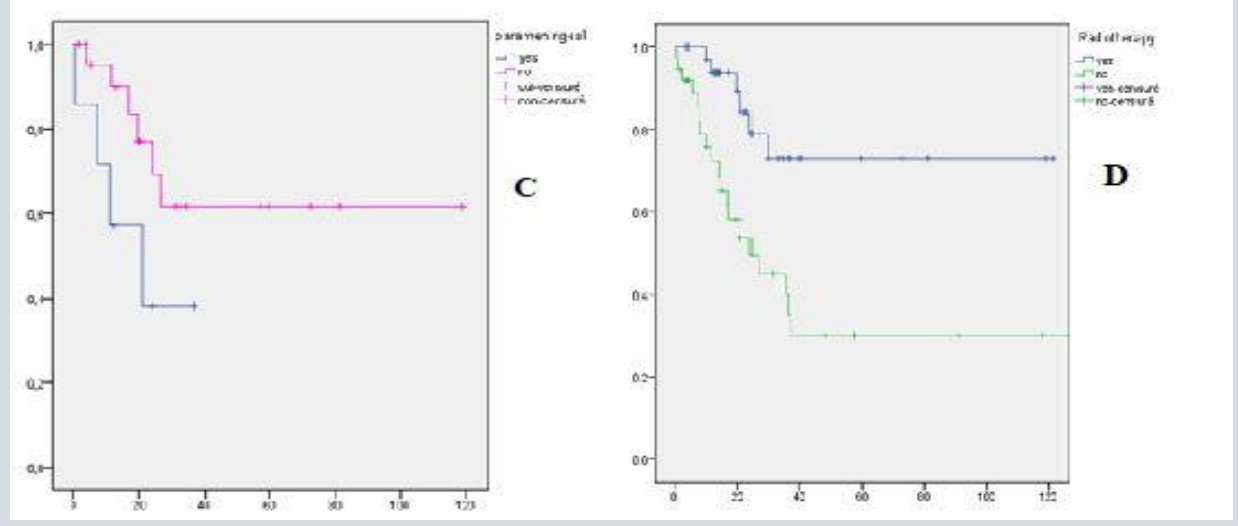

Figure 1: (A) Kaplan-Meier survival curves to histologic; (B) Size; (C): Para meningeal site;

(D) Radiotherapy.

Citation: Ksontini FL, et al. Non Metastatic Rhabdomyosarcoma in Children and Adolescents: Prognostic Factors and Survival. J Ortho Sci Res. 2021;2(3):1-10. 


\section{Discussion}

The treatment results in rhabdomyosarcoma has improved in the last 3 decades thanks to IRS studies as well as SIOP (International Society of Pediatric Oncology) studies. The overall longterm survival rate for such patients with non-metastatic disease is expected to exceed $80 \%$ [5]. The two major histological subtypes of RMS (alveolar and embryonal) have different clinical outcomes and prognostic factors [6].

Several studies had shown the unfavourable prognosis of alveolar RMS (RMSA) versus embryonal RMS (RMSE). Raney and al found a DFS $88 \%$ for RMSE versus $66 \%$ for RMSA. This substantial difference in prognosis between RMS types may be due to well-known distinct genetic alterations that putatively play a role in the pathogenesis of these tumors and their response to treatment. For example, RMSA presents a genetic alteration which generates protein fusions. So, we distinguish two types: FNRMS (Fusion Negative RMS) and FPRMS (Fusion Positive RMS). FNRMS has the same well prognosis as RMSE [6]. In Tunisia, we haven't yet molecular study, this can explain our results that alveolar RMS had a better overall survival may be almost of them were FNRMS.

Age at presentation is also an important prognostic indicator for survival of patients with RMS. Joshi, et al., classified patients into three failure-risk categories based on age ( $<1 \mathrm{y} ; 1-9 \mathrm{y} ;\rangle 10$ y) finding that infants and adolescents had significantly worse outcomes and thereby identifying patient age as an independent risk factor for treatment failure in RMS [7]. Our analysis failed to be significant.

Long-term outcome and treatment recommendations for RMS are based on multiple factors. Pretreatment staging is based on a site (favorable versus unfavorable) modified TNM system. Clinical group assignment is used following the initial surgical procedure and is primarily based on the surgical resect ability of the tumor, extent of residual tumor and presence of metastasis. Current COG protocols stratify patients into one of three risks treatment protocols according to tumor site, size, histology, TNM stage, clinical group and patient age [8]. Our analysis confirms only that that para meningeal site and tumor size have significant impact on survival for patients with RMS with a cut off $4 \mathrm{~cm}$. However, in the literature data, the cut off is $5 \mathrm{~cm}$.

Lymphs node status is an important part of pre-treatment staging, clinical group and impacts risk-based treatment strategies in RMS. Nodal disease is also incorporated into Clinical Group as determined by pathology. Lymph node involvement is present in $23 \%$ of all RMS patients, predominantly in primary sites such as retroperitoneum, perineum, extremity, bladder/prostate, paratesticular and para meningeal. Positive lymph nodes status is an independent poor prognostic factor for overall and disease free survival in patients with fusion positive ARMS but is not as important for fusion negative ERMS patients provided the nodal disease is treated appropriately with RT [9]. For our patients, lymph node positive RMS had a worse prognosis 
with an OS $40 \%$ versus $60 \%$ for N. But, it failed to be significant in multivariate analysis and for the DFS.

Surgery is the backbone of the treatment of RMS. Alone, it achieved a cure in $>20 \%$ of patients with RMS, which supposes that microscopic residual tumor invariably is remaining or disseminated in the majority of patients despite a total resection [8]. Survival has improved considerably, largely due to cooperative group trials (IRSG) and SIOP protocols employing multidisciplinary treatment protocols, which include multiagent chemotherapy and radiotherapy in addition to surgery. Surgical resection remains the standard treatment for localized disease as long as functional and/or cosmetic results are acceptable [9].

By univariate and multivariate analysis, our patients undergoing surgery hadn't significantly higher OS or DFS.

Radiotherapy is used in all patients with RMS except for those with localized embryonal tumors that are completely excised without residual disease. In advanced cases, radiation therapy plays a critical role in achieving local tumor control [10]. Overall, we found that radiation therapy was associated with an important survival benefit. Furthermore, adjuvant radiotherapy following surgery was associated with improved survival.

Currently, multiagent chemotherapy is indicated for all patients with RMS. Following surgical resection, combination therapy consisting of vincristine, dactinomycin and cyclophosphamide (VAC) or vincristine, dactinomycin and Ifosfamide (IVA) is administered to achieve eradication of microscopic residual disease [11,12]. Neoadjuvant chemotherapy can be indicated in case of unresectable tumors or to obtain cytoreduction and facilitate subsequent surgical excision. However, chemotherapy failed to improve survival in this study.

\section{Conclusion}

This single institutional study analysis showed that survival in paediatric patients with nonmetastatic rhabdomyosarcoma is low compared to litterature. Clinical trials which are focused on the oncogenic mechanisms of these tumours proposed new therapies. Unfortunately, these molecules are emerging slowly in low income countries such as Tunisia.

\section{References}

1. Koscielniak E, Harms D, Henze G, Jürgens H, Gadner H, Herbst M, et al. Results of treatment for soft tissue sarcoma in childhood and adolescence: a final report of the German cooperative soft tissue sarcoma study CWS-86. J Clin Oncol. 1999;17(12):3706-19.

2. Stevens MCG, Rey A, Bouvet N, Ellershaw C, Flamant F, Habrand JL, et al. Treatment of non-metastatic rhabdomyosarcoma in childhood and adolescence: third study of the international society of paediatric oncology-SIOP malignant mesenchymal tumor 89. J Clin Oncol. 2005;8:130. 
3. Crist W, Gehan EA, Ragab AH, Dickman PS, Donaldson SS, Fryer C, et al. The third intergroup rhabdomyosarcoma study. J Clin Oncol. 1995;13(3):610-30.

4. Rodary C, Rey A, Olive D, Flamant F, Quintana E, Brunat-Mentigny M, et al. Prognostic factors in 281 children with nonmetastatic Rhabdomyosarcoma (RMS) at diagnosis. Med Pediatr Oncol. 1988;16(2):71-7.

5. Perez E, Koniaris L, Neville H, Sola J. Rhabdomyosarcoma in children: A SEER population based study. J Surg Res. 2011;170:e243-51.

6. Meza JL, Anderson J, Pappo AS, Meyer WH. Analysis of prognostic factors in patients with nonmetastatic rhabdomyosarcoma treated on intergroup rhabdomyosarcoma studies III and IV: the Children's Oncology Group. J Clin Oncol. 2006;24(24):3844-51.

7. Davicioni E, Anderson MJ, Finckenstein FG, Lynch JC, Qualman SJ, Shimada H, et al. Molecular classification of Rhabdomyosarcoma-genotypic and phenotypic determinants of diagnosis. Am J Pathol. 2009;174(2):550-64.

8. Joshi VV, Balarezo F, Hicks JM, Mierau GW, Tsongalis GJ. Approach to small round cell tumors of childhood. AJSP Rev Rep. 2000;5(1):26-41.

9. Dasgupta R, Fuchs J, Rodeberg D. Rhabdomyosarcoma. Semin Pediatr Surg. 2016;25(5):276-83.

10. Michalski JM, Meza J, Breneman JC, Wolden SL, Laurie F, Jodoin MA, et al. Influence of radiation therapy parameters on outcome in children treated with radiation therapy for localized parameningeal rhabdomyosarcoma in Intergroup Rhabdomyosarcoma Study Group trials II through IV. Int J Radiat Oncol. 2004;59(4):1027-38.

11. Carli M, Colombatti R, Oberlin O, Bisogno G, Treuner J, Koscielniak E, et al. European intergroup studies (MMT4-89 and MMT4-91) on childhood metastatic rhabdomyosarcoma: final results and analysis of prognostic factors. J Clin Oncol. 2004;22(23):4787-94.

12. Crist WM, Anderson JR, Meza JL, Fryer C, Raney RB, Ruymann FB, et al. Intergroup Rhabdomyosarcoma study-IV: results for patients with nonmetastatic disease. J Clin Oncol. 2001;19(12):3091-102. 